\title{
THE BURGESS BOUND VIA A TRIVIAL DELTA METHOD
}

\author{
KESHAV AGGARWAL, ROMAN HOLOWINSKY, YONGXIAO LIN, AND QINGFENG SUN
}

\begin{abstract}
Let $g$ be a fixed Hecke cusp form for $\operatorname{SL}(2, \mathbb{Z})$ and $\chi$ be a primitive Dirichlet character of conductor $M$. The best known subconvex bound for $L(1 / 2, g \otimes \chi)$ is of Burgess strength. The bound was proved by a couple of methods: shifted convolution sums and the Petersson/Kuznetsov formula analysis. It is natural to ask what inputs are really needed to prove a Burgess-type bound on GL(2). In this paper, we give a new proof of the Burgess-type bounds $L(1 / 2, g \otimes \chi) \ll_{g, \varepsilon} M^{1 / 2-1 / 8+\varepsilon}$ and $L(1 / 2, \chi) \ll_{\varepsilon} M^{1 / 4-1 / 16+\varepsilon}$ that does not require the basic tools of the previous proofs and instead uses a trivial delta method.
\end{abstract}

\section{INTRODUCTION AND STATEMENT OF RESULTS}

Let $g$ be a fixed Hecke cusp form on GL(2) and let $\chi$ be a primitive Dirichlet character modulo $M$. Subconvex bounds for the twisted $L$-functions

$$
L(s, g \otimes \chi)=\sum_{n=1}^{\infty} \frac{\lambda_{g}(n) \chi(n)}{n^{s}}
$$

have been of interest for a while, with lots of applications. The strongest known bound is of Burgess quality (in special cases better results than Burgess-type exponent are known). The first subconvex bound in the conductor aspect was established by Burgess [4] for Dirichlet $L$-functions $L(s, \chi)$. For a primitive Dirichlet character $\chi$ modulo $M$, Burgess proved

$$
L\left(\frac{1}{2}, \chi\right) \ll_{\varepsilon} M^{1 / 4-1 / 16+\varepsilon} .
$$

A subconvex bound of such strength is called a Burgess bound. In the GL(2) setting, the Burgess bound for an $L$-function of a Hecke cusp form $g$ twisted by a primitive Dirichlet character $\chi$ of large conductor $M$ is

$$
L\left(\frac{1}{2}, g \otimes \chi\right) \ll_{g, \varepsilon} M^{1 / 2-1 / 8+\varepsilon} .
$$

The first subconvex bound for $L(1 / 2, g \otimes \chi)$ was obtained by Duke, Friedlander, and Iwaniec [7], who studied an amplified second moment and reduced the problem to treating shifted convolution sums of the form

$$
\sum_{\ell_{1} m-\ell_{2} n=h} \overline{\lambda_{g}(m)} \lambda_{g}(n),
$$

for which they invented the $\delta$-symbol method in their name to deal with. Their approach gives

$$
L\left(\frac{1}{2}, g \otimes \chi\right) \ll_{g, \varepsilon} M^{1 / 2-1 / 22+\varepsilon},
$$

where $g$ is a holomorphic cusp form on the full modular group. Bykovskiu [5], who embedded the $L$-function $L(1 / 2, g \otimes \chi)$ in question into a larger family $\sum_{g^{\prime} \in S(M)}\left|L\left(1 / 2, g^{\prime} \otimes \chi\right)\right|^{2}$ (and with amplification) and treated the later by applying the Petersson formula, where $S(M)$ is an orthogonal basis of the space of holomorphic cusp forms of level $M$, was able to sharpen the bound of Duke, Friedlander, and Iwaniec to the Burgess quality (2). Bykovskil's approach has the advantage of avoiding the treatment of shifted convolution sums and is regarded as the simplest subconvex proof for this case for a while. For more general cusp forms $g$ the bound (2) was established by Blomer, Harcos, and Michel [3] under the Ramanujan conjecture, and subsequently by Blomer and Harcos [2] unconditionally. This latter bound was generalized by Fouvry, Kowalski, and Michel $[8]$ to more general algebraic twists including the Dirichlet characters $\chi$.

2010 Mathematics Subject Classification. 11F66.

Key words and phrases. subconvexity, Dirichlet characters, Hecke cusp forms, $L$-functions. 
It is then natural to ask: what inputs are really needed to prove a Burgess-type bound on GL(2)? Is the shifted convolution sum treatment really needed? Is there anything special about the Petersson formula analysis? Or is subconvexity, even with the strongest known exponent, a soft feature? In asking these questions, we were able to find a proof that requires none of the tools required by the previous works. We came across this argument through a careful analysis of Munshi's GL(2) $\delta$-symbol proof in [18].

In a series of papers [16,17], Munshi introduced a novel GL(2) Petersson $\delta$-symbol method to prove level aspect subconvex bounds for GL(3) L-functions. In a recent paper [18], he demonstrated that the GL(2) $\delta$-symbol method can also be applied to the classical setting of Dirichlet $L$-functions as well as $L(s, g \otimes \chi)$ and re-established the bounds (1) and (2) simultaneously.

While studying the works of Munshi, Holowinsky and Nelson [10] discovered the following key identity hidden within Munshi's proof [17],

$$
\chi(n)=\frac{M}{R g_{\bar{\chi}}} \sum_{r=1}^{\infty} \chi(r) e\left(\frac{n \bar{r}}{M}\right) V\left(\frac{r}{R}\right)-\frac{1}{g_{\bar{\chi}}} \sum_{r \neq 0} S_{\chi}(r, n ; M) \hat{V}\left(\frac{r}{M / R}\right) .
$$

Here $g_{\chi}$ is the Gauss sum, $S_{\chi}(r, n ; M)$ is the generalized Kloosterman sum, $\hat{V}$ is the Fourier transform of the Schwartz function $V$ which is supported on $[1,2]$ and is normalized such that $\hat{V}(0)=1$, and $R>0$ is a parameter. This allowed them to produce a method which removed the use of the GL(2) $\delta$-symbol and establish a stronger subconvex bound. Subsequently, Lin [12] was able to generalize the identity in the application to the subconvexity problem in both the Dirichlet character twist and $t$-aspect case via the identity

$$
\begin{aligned}
\chi(n) n^{-i t} V_{A}\left(\frac{n}{N}\right) & =O\left(t^{1 / 2-A}\right)+\left(\frac{2 \pi}{M t}\right)^{i t} e\left(\frac{t}{2 \pi}\right) \frac{M^{2} t^{3 / 2}}{N g_{\bar{\chi}}} \sum_{r=1}^{\infty} \chi(r) e\left(\frac{n \bar{r}}{M}\right) r^{-i t} e\left(-\frac{n}{M r}\right) V\left(\frac{r}{N / M t}\right) \\
& -\left(\frac{2 \pi}{N}\right)^{i t} e\left(\frac{t}{2 \pi}\right) \frac{t^{1 / 2}}{g_{\bar{\chi}}} \sum_{r \neq 0} S_{\chi}(r, n ; M) \int_{\mathbb{R}} V(x) x^{-i t} e\left(-\frac{n t}{N x}\right) e\left(-\frac{r N x}{M^{2} t}\right) \mathrm{d} x .
\end{aligned}
$$

Here $n=N, A \geqslant 1$ is any integer, $V_{A}(x)$ is a smooth compactly supported function with bounded derivatives. With this approach, Lin [12] obtained the following bound

$$
L\left(\frac{1}{2}+i t, \pi \otimes \chi\right) \ll_{\pi, \varepsilon}(M(|t|+1))^{3 / 4-1 / 36+\varepsilon},
$$

for $\pi$ being a fixed Hecke-Maass cusp form for $\mathrm{SL}(3, \mathbb{Z})$.

In this paper, we demonstrate that one is again able to remove the GL(2) $\delta$-symbol method and replace it in our subconvexity problem by the following trivial key identity,

$$
\delta(n=0)=\frac{1}{q} \sum_{c \mid q} \sum_{\substack{a \bmod c \\(a, c)=1}} e\left(\frac{a n}{c}\right), \text { when } q>|n|,
$$

where $\delta(n=0)$ denotes the Kronecker delta symbol. We shall establish the following bounds.

Theorem 1.1. Let $g$ be a fixed Hecke cusp form for $\mathrm{SL}(2, \mathbb{Z})$ and $\chi$ be a primitive Dirichlet character modulo a prime $M$. For any $\varepsilon>0$,

$$
L\left(\frac{1}{2}, g \otimes \chi\right) \ll_{g, \varepsilon} M^{1 / 2-1 / 8+\varepsilon} .
$$

Theorem 1.2. Let $\chi$ be a primitive Dirichlet character modulo a prime $M$. For any $\varepsilon>0$,

$$
L\left(\frac{1}{2}, \chi\right) \ll_{\varepsilon} M^{1 / 4-1 / 16+\varepsilon} .
$$

Our approach is more direct. Neither the treatment of shifted convolution sums nor the use of the Petersson/Kuznetsov formula, is needed. We should point out that in Burgess' proof in [4] he appealed to estimates from algebraic geometry, which follow from Weil's proof of the Riemann hypothesis for curves over finite fields. In our case, the algebraic geometry input that we use is the work [1] of Adolphson and Sperber who proved their results in light of Deligne's general results. We also remark that better bounds were known for $\chi$ quadratic: Conrey and Iwaniec [6] proved that the Weyl-type bound $L\left(\frac{1}{2}, \chi\right) \ll_{\varepsilon} M^{1 / 6+\varepsilon}$ holds. This bound was recently extended to any character $\chi$ by Petrow and Young $[19,20]$. 


\section{Some Notations And Lemmas}

For a smooth function $V$ with bounded derivatives, we define its Fourier transform

$$
\widehat{V}(x)=\int_{\mathbb{R}} V(u) e(-x u) \mathrm{d} u .
$$

Repeated integration by parts shows

$$
\widehat{V}(x) \ll_{A}(1+|x|)^{-A}
$$

for any $A \geqslant 0$.

Next, we collect some lemmas that we will use for the proof.

Lemma 2.1 (Trivial delta method). One has

$$
\delta(n \equiv m \bmod q)=\frac{1}{q} \sum_{c \mid q} \sum_{a \bmod c}^{\star} e\left(\frac{a(n-m)}{c}\right) .
$$

where the star over the inner sum denotes the sum is over $(a, c)=1$.

Lemma 2.2 (Voronoi summation formula, [11, Theorem A.4]). Let $g$ be a Hecke cusp form of level 1 with Fourier coefficients $\lambda_{g}(n)$. Let $c \in \mathbb{N}$ and $a \in \mathbb{Z}$ be such that $(a, c)=1$ and let $W$ be a smooth compactly supported function. For $N>0$,

$$
\sum_{n=1}^{\infty} \lambda_{g}(n) e\left(\frac{a n}{c}\right) W\left(\frac{n}{N}\right)=\mathcal{I}(g ; W, c, N)+\frac{N}{c} \sum_{ \pm} \sum_{n=1}^{\infty} \lambda_{g}(n) e\left(\mp \frac{\bar{a} n}{c}\right) \widetilde{W}_{g}^{ \pm}\left(\frac{n N}{c^{2}}\right),
$$

where

$$
\mathcal{I}(g ; W, c, N)=\left\{\begin{array}{cl}
\frac{N}{c} \int_{0}^{\infty}(\log x N+2 \gamma-2 \log c) W(x) \mathrm{d} x & \text { if } \lambda_{g} \text { is the divisor function } \tau, \\
0 & \text { otherwise. }
\end{array}\right.
$$

Here $\gamma$ is the Euler's constant. $\widetilde{W}_{g}^{ \pm}$is an integral transform of $W$ given by the following.

(1) If $g$ is holomorphic of weight $k$, then

$$
\widetilde{W}_{g}^{+}(y)=\int_{0}^{\infty} W(x) 2 \pi i^{k} J_{k-1}(4 \pi \sqrt{y x}) \mathrm{d} x,
$$

and $\widetilde{W}_{g}^{-}=0$.

(2) If $g$ is a Maass form with $(\Delta+\lambda) g=0$ and $\lambda=1 / 4+r^{2}$, and $\varepsilon_{g}$ is an eigenvalue under the reflection operator,

$$
\widetilde{W}_{g}^{+}(y)=\int_{0}^{\infty} \frac{-\pi W(x)}{\sin \pi i r}\left(J_{2 i r}(4 \pi \sqrt{y x})-J_{-2 i r}(4 \pi \sqrt{y x})\right) \mathrm{d} x,
$$

and

$$
\widetilde{W}_{g}^{-}(y)=\int_{0}^{\infty} 4 \varepsilon_{g} \cosh (\pi r) W(x) K_{2 i r}(4 \pi \sqrt{y x}) \mathrm{d} x .
$$

If $r=0$,

$$
\widetilde{W}_{g}^{+}(y)=\int_{0}^{\infty}-2 \pi W(x) Y_{0}(4 \pi \sqrt{y x}) \mathrm{d} x \quad \text { and } \quad \widetilde{W}_{g}^{-}(y)=\int_{0}^{\infty} 4 \varepsilon_{g} W(x) K_{0}(4 \pi \sqrt{y x}) \mathrm{d} x .
$$

(3) When $\lambda_{g}(n)=\tau(n)$ is the divisor function,

$$
\widetilde{W}_{g}^{+}(y)=\int_{0}^{\infty}-2 \pi Y_{0}(4 \pi \sqrt{x y}) \mathrm{d} x \quad \text { and } \quad \widetilde{W}_{g}^{-}(y)=\int_{0}^{\infty} 4 K_{0}(4 \pi \sqrt{y x}) \mathrm{d} x .
$$

In each case, we have

$$
\widetilde{W}_{g}^{ \pm}(x) \ll_{A}(1+|x|)^{-A},
$$

for any $A \geqslant 0$.

Very often we will use the following bound when $g$ is a Maass form with Fourier coefficients $\lambda_{g}(n)$. 
Lemma 2.3 (Ramanujan bound on average). Let $W$ be a smooth function with compact support contained in $\mathbb{R}_{>0}$, satisfying $W^{(j)}(x) \ll_{j} 1$. Then

$$
\sum_{n=1}^{\infty}\left|\lambda_{g}(n)\right| W\left(\frac{n}{X}\right) \ll_{g} X
$$

This follows from the Cauchy-Schwarz inequality and the Rankin-Selberg estimate $\sum_{n \leqslant X}\left|\lambda_{g}(n)\right|^{2} \ll_{g} X$ (see [15]).

\section{Sketch OF THE PROOF}

Since there are quite a few auxiliary parameters involved in our proof, some are essential while others are not, in this section we provide a quick sketch to guide the reader through the essential part of our argument.

For $\chi \bmod M$, we start with the following sum

$$
S(N):=\sum_{n \sim N} \lambda_{g}(n) \chi(n)
$$

where the sum is interpreted with a smooth function which controls the support being attached. Through out this section we will not display the smooth test functions in each transformations. We will focus on the generic term, ignoring various error terms whose contributions are not essential. Our goal is to beat the convexity bound $O(N)$ of $S(N)$.

We let $P, L \gg 1$ be two parameters such that $P M \gg N L$. By using the Hecke relation $\lambda_{g}(n \ell) \approx \lambda_{g}(n) \lambda_{g}(\ell)$ and the approximation

$$
\delta(n, r \ell) \approx \frac{1}{p M} \sum_{\alpha(p M)}^{\star} e\left(\frac{\alpha(n-r \ell)}{p M}\right)
$$

we can write

$$
\begin{aligned}
S(N) & =\frac{1}{L} \sum_{\ell \sim L} \overline{\lambda_{g}(\ell)} \sum_{n \sim N \ell} \lambda_{g}(n) \sum_{r \sim N} \chi(r) \delta(n, r \ell) \\
& \approx \frac{1}{L} \sum_{\ell \sim L} \overline{\lambda_{g}(\ell)} \sum_{n \sim N L} \lambda_{g}(n) \sum_{r \sim N} \chi(r) \frac{1}{P} \sum_{p \sim P} \frac{1}{p M} \sum_{\alpha(p M)}^{\star} e\left(\frac{\alpha(n-r \ell)}{p M}\right) \\
& \approx \frac{1}{P^{2} M L} \sum_{p \sim P} \sum_{\ell \sim L} \overline{\lambda_{g}(\ell)} \sum_{\alpha(p M)}^{\star} \sum_{n \sim N L} \lambda_{g}(n) e\left(\frac{\alpha n}{p M}\right) \sum_{r \sim N} \chi(r) e\left(\frac{-\alpha r \ell}{p M}\right) .
\end{aligned}
$$

Here $p \sim P$ and $\ell \sim L$ denote primes in the dyadic intervals $[P, 2 P]$ and $[L, 2 L]$.

We then dualize the $n$ and $r$ sums using Voronoi summation and Poisson summation, respectively, getting

$$
\sum_{n \sim N L} \lambda_{g}(n) e\left(\frac{\alpha n}{p M}\right) \leftrightarrow \frac{N L}{p M} \sum_{n \sim \frac{p^{2} M^{2}}{N L}} \overline{\lambda_{g}(n)} e\left(\frac{-\bar{\alpha} n}{p M}\right)
$$

and

$$
\begin{aligned}
\sum_{r \sim N} \chi(r) e\left(\frac{-\alpha r \ell}{p M}\right) & \leftrightarrow \frac{N}{p M} \sum_{r<\frac{P M}{N}} \sum_{\beta(p M)} \chi(\beta) e\left(\frac{-\alpha \beta \ell}{p M}\right) e\left(\frac{\beta r}{p M}\right) \\
& =\frac{N}{p M} \sum_{r<\frac{P M}{N}} g_{\chi} \chi(p) \bar{\chi}(r-\alpha \ell) \cdot p \delta_{r \equiv \alpha \ell \bmod p} .
\end{aligned}
$$


By splitting $\sum_{\alpha(p M)}^{\star}$ into $\sum_{\alpha(M)}^{\star}$ and $\sum_{\alpha(p)}^{\star}$ and putting things together, we get

$$
\begin{aligned}
S(N)= & \frac{1}{P^{2} M L} \sum_{p \sim P} \sum_{\ell \sim L} \overline{\lambda_{g}(\ell)} \frac{N L}{p M} \sum_{n \sim \frac{P^{2} M^{2}}{N L}} \overline{\lambda_{g}(n)} \\
& \cdot \frac{N}{p M} \sum_{r<\frac{P M}{N}}\left(\sum_{\alpha(M)}^{\star} e\left(\frac{-\bar{\alpha} n \bar{p}}{M}\right) g_{\chi} \chi(p) \bar{\chi}(r-\alpha \ell)\right) \cdot \sum_{\alpha(p)}^{\star}\left(e\left(\frac{-\bar{\alpha} n \bar{M}}{p}\right) p \delta_{r \equiv \alpha \ell \bmod p)}\right. \\
\approx & \frac{N^{2}}{P^{3} M^{5 / 2}} \sum_{n \sim \frac{P^{2} M^{2}}{N L}} \overline{\lambda_{g}(n)} \sum_{p \sim P} \chi(p) \sum_{\ell \sim L} \overline{\lambda_{g}(\ell)} \sum_{r<\frac{P M}{N}} e\left(\frac{-\bar{r} n \ell \bar{M}}{p}\right) \sum_{\alpha(M)}^{\star} e\left(\frac{-\bar{\alpha} n \ell \bar{p}}{M}\right) \bar{\chi}(r-\alpha) .
\end{aligned}
$$

We then use Cauchy-Schwarz to remove the GL(2) coefficients $\overline{\lambda_{g}(n)}$ and get

$$
\begin{aligned}
S(N) \ll & \frac{N^{2}}{P^{3} M^{5 / 2}}\left(\sum_{n \sim \frac{P^{2} M^{2}}{N L}}\left|\overline{\lambda_{g}(n)}\right|^{2}\right)^{1 / 2} \\
& \left(\sum_{n \sim \frac{P^{2} M^{2}}{N L}}\left|\sum_{p \sim P} \chi(p) \sum_{\ell \sim L} \overline{\lambda_{g}(\ell)} \sum_{r<\frac{P M}{N}} e\left(\frac{-\bar{r} n \ell \bar{M}}{p}\right) \sum_{\alpha(M)}^{\star} e\left(\frac{-\bar{\alpha} n \ell \bar{p}}{M}\right) \bar{\chi}(r-\alpha)\right|^{2}\right)^{1 / 2} .
\end{aligned}
$$

Remark 3.1. The contribution from the "diagonal term" $\left(p_{1}, \ell_{1}, r_{1}, \alpha_{1}\right)=\left(p_{2}, \ell_{2}, r_{2}, \alpha_{2}\right)$ is given by

$$
S_{\text {diag }} \ll \frac{N^{2}}{P^{3} M^{5 / 2}} \frac{P^{2} M^{2}}{N L}\left(P L \frac{P M}{N} M\right)^{1 / 2} \ll \frac{N^{1 / 2} M^{1 / 2}}{L^{1 / 2}},
$$

which improves over the trivial bound $O(M)$ as long as $L$ has some size.

Opening the absolute valued square and switching the order of summations, we get

$$
\begin{gathered}
S(N) \ll \frac{N^{3 / 2}}{P^{2} M^{3 / 2} L^{1 / 2}}\left(\sum_{p_{1}, p_{2}} \chi\left(p_{1}\right) \bar{\chi}\left(p_{2}\right) \sum_{\ell_{1}, \ell_{2}} \overline{\lambda_{g}\left(\ell_{1}\right)} \lambda_{g}\left(\ell_{2}\right) \sum_{r_{1}<\frac{P M}{N}} \sum_{r_{2}<\frac{P M}{N} \alpha_{1}(M) \alpha_{2}(M)}^{\star} \sum_{n \sim \frac{P^{2} M^{2}}{N L}}^{\star} \bar{\chi}\left(\frac{\left.-r_{1}-\alpha_{1}\right) \chi\left(r_{2}-\alpha_{2}\right)}{p_{1}}+\frac{\overline{r_{2}} n \ell_{2} \bar{M}}{p_{2}}\right) e\left(\frac{-\overline{\alpha_{1}} n \ell_{1} \overline{p_{1}}}{M}+\frac{\overline{\alpha_{2}} n \ell_{2} \overline{p_{2}}}{M}\right)\right)^{1 / 2} . \\
\sum .
\end{gathered}
$$

The $n$-sum in the second line above, by applying Poisson summation, is

$$
\begin{aligned}
\sum_{n \sim \frac{P^{2} M^{2}}{N L}}(\cdots) \leftrightarrow & \frac{P^{2} M^{2}}{N L} \frac{1}{p_{1} p_{2} M} \sum_{n \ll \frac{p_{1} p_{2} M}{P^{2} M^{2} / N L}} \sum_{\beta\left(p_{1} p_{2}\right)} e\left(\frac{\beta\left(-\overline{r_{1}} \ell_{1} p_{2}+\overline{r_{2}} \ell_{2} p_{1}+n\right) \bar{M}}{p_{1} p_{2}}\right) \\
& \sum_{\gamma(M)} e\left(\frac{\gamma\left(-\overline{\alpha_{1}} \ell_{1} \overline{p_{1}}+\overline{\alpha_{2}} \ell_{2} \overline{p_{2}}+n \overline{p_{1} p_{2}}\right)}{M}\right) \\
= & \frac{P^{2} M^{2}}{N L} \sum_{n \ll \frac{N L}{M}} \delta_{-\overline{r_{1}} \ell_{1} p_{2}+\overline{r_{2}} \ell_{2} p_{1}+n \equiv 0 \bmod p_{1} p_{2}} \cdot \delta_{\alpha_{2} \equiv \ell_{2} p_{1} \overline{\overline{\alpha_{1}} \ell_{1} p_{2}-n} \bmod M .} .
\end{aligned}
$$

Plugging this back to the previous estimate, we obtain

$$
\begin{aligned}
S(N) \ll & \frac{N}{P M^{1 / 2} L} \mid \sum_{p_{1}, p_{2}} \chi\left(p_{1}\right) \bar{\chi}\left(p_{2}\right) \sum_{\ell_{1}, \ell_{2}} \overline{\lambda_{g}\left(\ell_{1}\right)} \lambda_{g}\left(\ell_{2}\right) \sum_{r_{1}, r_{2}<\frac{P M}{N}} \sum_{n \ll \frac{N L}{M}} \delta_{-\overline{r_{1}} \ell_{1} p_{2}+\overline{r_{2}} \ell_{2} p_{1}+n \equiv 0 \bmod p_{1} p_{2}} \\
& \left.\cdot \sum_{\alpha(M)}^{\star} \bar{\chi}\left(r_{1}-\alpha\right) \chi\left(r_{2}-\ell_{2} p_{1} \overline{\bar{\alpha} \ell_{1} p_{2}-n}\right)\right|^{1 / 2} .
\end{aligned}
$$

The contribution from the zero-frequency $n=0$ will essentially correspond to the diagonal contribution

$$
S_{\text {diag }} \ll \frac{N^{1 / 2} M^{1 / 2}}{L^{1 / 2}}
$$


in Remark 3.1.

For $n \neq 0$, assuming the parameters $\left(p_{1}, \ell_{1}, r_{1}, p_{2}, \ell_{2}, r_{2}\right)$ are in the generic position, then by appealing to Weil's theory, one has

$$
\frac{1}{M^{1 / 2}} \sum_{\alpha(M)}^{\star} \bar{\chi}\left(r_{1}-\alpha\right) \chi\left(r_{2}-\ell_{2} p_{1} \overline{\bar{\alpha} \ell_{1} p_{2}-n}\right) \ll 1
$$

Hence the contribution $S_{\text {off }}$ from the non-zero frequencies $n \neq 0$ is given by

$$
S_{\text {off }} \ll \frac{N}{P M^{1 / 2} L}\left(P^{2} L^{2} \frac{P M}{N} \frac{P M}{N} \frac{N L}{M} M^{1 / 2} \frac{1}{P^{2}}\right)^{1 / 2} \ll N^{1 / 2} M^{1 / 4} L^{1 / 2} .
$$

In conclusion, we obtain

$$
S(N) \ll S_{\text {diag }}+S_{\text {off }} \ll \frac{N^{1 / 2} M^{1 / 2}}{L^{1 / 2}}+N^{1 / 2} M^{1 / 4} L^{1 / 2} .
$$

By choosing $L=M^{1 / 4}$ and $P>\frac{N}{M^{3 / 4}}$ we obtain

$$
S(N) \ll N^{1 / 2} M^{3 / 8}
$$

which will imply the Burgess bound as stated in Theorem 1.1.

Remark 3.2. One can interpret the left hand side of (8) as a Frobenius trace function $K(n)$ (depending on $\left.\left(p_{1}, \ell_{1}, r_{1}, p_{2}, \ell_{2}, r_{2}\right)\right)$ modulo $M$. In order to improve the Burgess bound, one might think to improve the bound (8) "on average", by taking advantage of the extra summation over $n$ in (7). But this is difficult since the length of the summation is below the Polyá-Vinogradov range.

\section{The SET-UP}

For any $N \geqslant 1$, define the following sum

$$
S(N)=\sum_{n=1}^{\infty} \lambda_{g}(n) \chi(n) W\left(\frac{n}{N}\right)
$$

where $W$ is a smooth bump function supported on [1,2] with $W^{(j)}(x) \ll_{j} 1$. Estimating the sum trivially with (6) gives the bound $S(N) \ll_{\varepsilon} N^{1+\varepsilon}$. Using an approximate functional equation of $L(s, g \otimes \chi)$, one can derive the following.

Lemma 4.1. For any $0<\delta<1$, we have

$$
L\left(\frac{1}{2}, g \otimes \chi\right) \ll M^{\varepsilon} \sup _{N} \frac{|S(N)|}{\sqrt{N}}+M^{1 / 2-\delta / 2+\varepsilon},
$$

where the supremum is taken over $N$ in the range $M^{1-\delta}<N<M^{1+\varepsilon}$.

From the above lemma, it suffices to improve the bound $S(N) \ll_{\varepsilon} N^{1+\varepsilon}$ in the range $M^{1-\delta}<N<M^{1+\varepsilon}$, where $\delta>0$ is a constant to be chosen later.

Let $\mathcal{L}$ be the set of primes $\ell$ in the dyadic interval $[L, 2 L]$, where $L<M^{1-\varepsilon}$ is a parameter to be determined later. Denote $L^{\star}=\sum_{\ell \in \mathcal{L}}\left|\lambda_{g}(\ell)\right|^{2}$. Then $L^{\star} \asymp \frac{L}{\log L}$, as the following argument shows. For $\varepsilon>0$,

$$
\sum_{\ell \in \mathcal{L}}\left|\lambda_{g}(\ell)\right|^{2} \asymp \frac{1}{\log L} \sum_{\ell \in \mathcal{L}}(\log \ell)\left|\lambda_{g}(\ell)\right|^{2}=\frac{1}{\log L} \sum_{n=L}^{2 L} \Lambda(n)\left|\lambda_{g}(n)\right|^{2}+O\left(L^{1-\varepsilon}\right),
$$

where $\Lambda(n)$ is the Von Mangoldt function. By the prime number theorem for automorphic representations (see [14, Corollary 1.2]), we have $\sum_{L \leqslant n \leqslant 2 L} \Lambda(n)\left|\lambda_{g}(n)\right|^{2} \sim L$. Thus

$$
L^{\star}=\sum_{\ell \in \mathcal{L}}\left|\lambda_{g}(\ell)\right|^{2} \asymp \frac{L}{\log L} .
$$

Similarly, we let $P$ be a parameter and $\mathcal{P}$ be the set of primes $p$ in the dyadic interval $[P, 2 P]$. Denote $P^{\star}=\sum_{p \in \mathcal{P}} 1 \asymp \frac{P}{\log P}$. We will choose $P$ and $L$ so that $\mathcal{P} \cap \mathcal{L}=\varnothing$. 
Let $p \in \mathcal{P}, n=N L$ and $r=N$. For $\varepsilon>0$ and $P M \gg(N L)^{1+\varepsilon}$, the condition $n=r \ell$ is equivalent to the congruence $n \equiv r \ell \bmod p M$. Since $N<M^{1+\varepsilon}$, we assume that,

$$
P \gg L^{1+\varepsilon} \text {. }
$$

Therefore, under the assumption

$$
P M \gg(N L)^{1+\varepsilon},
$$

by using the detection (4) with $q=p M$, the main sum of interest $S(N)$ defined in (9) can be expressed as

$$
\begin{aligned}
S(N)=\frac{1}{L^{\star}} \sum_{\ell \in \mathcal{L}} \overline{\lambda_{g}(\ell)} \sum_{n=1}^{\infty} \lambda_{g}(n) W\left(\frac{n}{N \ell}\right) \sum_{r=1}^{\infty} \chi(r) V\left(\frac{r}{N}\right) \delta(n=r \ell)\left(\frac{n}{r \ell}\right)^{i v}+O\left(\frac{N^{1+\varepsilon}}{L}\right) \\
=\frac{1}{L^{\star} P^{\star}} \sum_{\ell \in \mathcal{L}} \overline{\lambda_{g}(\ell)} \sum_{p \in \mathcal{P}} \frac{1}{p M} \sum_{c \mid p M} \sum_{\alpha \bmod c n=1}^{\star} \sum_{n}^{\infty} \lambda_{g}(n) e\left(\frac{\alpha n}{c}\right)\left(\frac{n}{N \ell}\right)^{i v} W\left(\frac{n}{N \ell}\right) \\
\times \sum_{r=1}^{\infty} \chi(r) e\left(\frac{-\alpha r \ell}{c}\right)\left(\frac{r}{N}\right)^{-i v} V\left(\frac{r}{N}\right)+O\left(\frac{N^{1+\varepsilon}}{L}\right) .
\end{aligned}
$$

Here $V$ is a smooth function supported on [1/2,3], constantly 1 on $[1,2]$ and satisfies $V^{(j)}(x) \ll_{j} 1$, and $\overline{\lambda_{g}(\ell)}$ denotes $\tau(\ell)^{-1}$ when $\lambda_{g}$ is the divisor function $\tau$, and

$$
v:=M^{\varepsilon} .
$$

The error term $O\left(N^{1+\varepsilon} L^{-1}\right)$ arises from the Hecke relation $\lambda_{g}(r \ell)=\lambda_{g}(r) \lambda_{g}(\ell)-\delta_{\ell \mid r} \lambda_{g}(1) \lambda_{g}(r / \ell)$.

Remark 4.2. (1) The point of introducing the extra $(n / r \ell)^{i v}$ factor with $v=M^{\varepsilon}$ is to insure that, after applying Voronoi and Poisson summations, in (15) the dual $n$ and $r$ summations are essentially supported on dyadic intervals, which would help simplify the subsequent counting arguments.

(2) The extra sum over $\ell$ is reminiscent of the amplification technique in [7] (see also [10,12,18]). Without introducing it we will be at the threshold to beat the convexity bound (cf. Remark 3.1).

Our strategy then is to apply dual summation formulas to the $n$ and $r$-sums, followed by applications of Cauchy-Schwarz inequality and Poisson summation to the $n$-sum. A careful analysis of the resulting congruence conditions together with Deligne's theory of exponential sums yields the final bounds.

\section{Application of DUAl SUmmation FORMulas}

We start with an application of the Voronoi summation formula (Lemma 2.2) to the $n$-sum in equation (11). Then,

$$
\begin{aligned}
S(N)=\mathcal{I}_{g=\tau}+\frac{N}{L^{\star} P^{\star}} \sum_{ \pm} \sum_{\ell \in \mathcal{L}} & \overline{\lambda_{g}(\ell)} \ell \sum_{p \in \mathcal{P}} \frac{1}{p M} \sum_{c \mid p M} \frac{1}{c} \sum_{n=1}^{\infty} \lambda_{g}(n) \widetilde{W}_{v, g}^{ \pm}\left(\frac{n}{c^{2} / N \ell}\right) \\
& \times \sum_{r=1}^{\infty} \chi(r) S(r \ell, \pm n ; c)\left(\frac{r}{N}\right)^{-i v} V\left(\frac{r}{N}\right)+O\left(\frac{N^{1+\varepsilon}}{L}\right),
\end{aligned}
$$

where $\mathcal{I}_{g=\tau}$ vanishes if $g$ is a cusp form, otherwise it is given by

$$
\begin{aligned}
& \mathcal{I}_{g=\tau}=\frac{N}{L^{\star} P^{\star}} \sum_{\ell \in \mathcal{L}} \overline{\lambda_{g}(\ell)} \ell \sum_{p \in \mathcal{P}} \frac{1}{p M} \sum_{c \mid p M} \frac{1}{c} \sum_{\alpha \bmod }^{\star} \sum_{r=1}^{\infty} \chi(r) e\left(\frac{-\alpha r \ell}{c}\right)\left(\frac{r}{N}\right)^{-i v} V\left(\frac{r}{N}\right) \\
& \times \int_{0}^{\infty}(\log x+2 \gamma+\log N \ell-2 \log c) W_{v}(x) \mathrm{d} x .
\end{aligned}
$$

Here $\widetilde{W_{v, g}^{ \pm}}$denotes the Hankel transform of the function $W_{v}(y):=y^{i v} W(y)$, defined in Lemma 2.2. By performing a stationary phase argument, the function $\widetilde{W}_{v, g}^{ \pm}(x)$ is negligibly small, unless $x=v^{2}=M^{2 \varepsilon}$ (for a proof see [13, Lemma 3.3]). Hence the $n$-variable in (13) is supported on $n=\frac{c^{2} v^{2}}{N \ell}$.

Next, we apply Poisson summation to the $r$-sums in (13). We introduce a few notations. For $a, b \in \mathbb{Z}$, we let $[a, b]$ be the lcm of $a$ and $b$; let $(a, b)$ be the gcd of $a$ and $b$, and let $a_{b}=a /(a, b)$. We note that if $a$ is squarefree, then $\left(b, a_{b}\right)=1$. 
Writing $S(r \ell, \pm n ; c)=\sum_{\alpha \bmod c}^{\star} e\left(\frac{\mp \bar{\alpha} n-\alpha r \ell}{c}\right)$, the $r$-sums in (13) are given by

$$
\sum_{r \geqslant 1} \chi(r) e\left(\frac{-\alpha r \ell}{c}\right)\left(\frac{r}{N}\right)^{-i v} V\left(\frac{r}{N}\right)
$$

Breaking the sum modulo $[c, M]$ and applying the Poisson summation formula, the $r$-sum becomes

$$
\frac{N}{[c, M]} \sum_{r \in \mathbb{Z}}\left(\sum_{\beta \bmod [c, M]} \chi(\beta) e\left(\frac{-\alpha \beta \ell}{c}\right) e\left(\frac{r \beta}{[c, M]}\right)\right) \widehat{V_{v}}\left(\frac{r N}{[c, M]}\right),
$$

where $V_{v}(y):=y^{-i v} V(y)$ and $\widehat{V_{v}}$ denotes the Fourier transform of $V_{v}$.

Using the relation $[c, M]=M c_{M}$ and reciprocity, the $\beta$-sum can be rewritten as

$$
\begin{aligned}
& \sum_{\beta \bmod M} \chi(\beta) e\left(\frac{\left(r-\alpha \ell M_{c}\right) \overline{c_{M}} \beta}{M}\right) \times \sum_{\beta \bmod c_{M}} e\left(\frac{\left(r-\alpha \ell M_{c}\right) \bar{M} \beta}{c_{M}}\right) \\
= & \bar{\chi}\left(\left(r-\alpha \ell M_{c}\right) \overline{c_{M}}\right) g_{\chi} \times c_{M} \delta\left(r-\alpha \ell M_{c} \equiv 0 \bmod c_{M}\right),
\end{aligned}
$$

where $g_{\chi}$ is the Gauss sum. By (3), one can truncate the $r$-sum at $|r| \ll[c, M] N^{\varepsilon} / N$, up to a negligible error. Therefore (14) becomes

$$
\frac{N g_{\chi}}{M} \sum_{\substack{|r| \ll[c, M] N^{\varepsilon} / N \\ r-\alpha \ell M_{c} \equiv 0 \bmod c_{M}}} \bar{\chi}\left(\left(r-\alpha \ell M_{c}\right) \overline{c_{M}}\right) \widehat{V_{v}}\left(\frac{r N}{[c, M]}\right)+O\left(N^{-2018}\right) .
$$

Substituting the above expression into (13), we arrive at

$$
\begin{gathered}
S(N)=\mathcal{M}_{g=\tau}+\frac{N^{2} g_{\chi}}{M L^{\star} P^{\star}} \sum_{ \pm} \sum_{\ell \in \mathcal{L}} \overline{\lambda_{g}(\ell)} \ell \sum_{p \in \mathcal{P}} \frac{1}{p M} \sum_{c \mid p M} \frac{1}{c} \sum_{\alpha \bmod }^{\star} \sum_{c=1}^{\infty} \lambda_{g}(n) e\left(\mp \frac{\bar{\alpha} n}{c}\right) \widetilde{W_{v}^{ \pm}, g}\left(\frac{n}{c^{2} / N \ell}\right) \\
\sum_{\substack{|r| \ll[c, M] N^{\varepsilon} / N \\
r-\alpha \ell M_{c} \equiv 0 \bmod c_{M}}} \bar{\chi}\left(\left(r-\alpha \ell M_{c}\right) \overline{c_{M}}\right) \widehat{V_{v}}\left(\frac{r N}{[c, M]}\right)+O\left(\frac{N^{1+\varepsilon}}{L}\right) .
\end{gathered}
$$

The term $\mathcal{M}_{g=\tau}$ vanishes if $g$ is a cusp form. Otherwise it is given by

$$
\begin{aligned}
\mathcal{M}_{g=\tau}= & \frac{N^{2} g_{\chi}}{M L^{\star} P^{\star}} \sum_{\ell \in \mathcal{L}} \overline{\lambda_{g}(\ell)} \ell \sum_{p \in \mathcal{P}} \frac{1}{p M} \sum_{c \mid p M} \frac{1}{c} \sum_{\alpha \bmod c}^{\star} \sum_{\substack{|r| \ll[c, M] N^{\varepsilon} / N \\
r-\alpha \ell M_{c} \equiv 0 \bmod c_{M}}} \bar{\chi}\left(\left(r-\alpha \ell M_{c}\right) \overline{c_{M}}\right) \widehat{V_{v}}\left(\frac{r N}{[c, M]}\right) \\
& \quad \times \int_{0}^{\infty}(\log x+2 \gamma+\log N \ell-2 \log c) W_{v}(x) \mathrm{d} x \\
& \ll \frac{N(P M)^{\varepsilon}}{\sqrt{M} L P} \sum_{1 \leqslant \ell \leqslant 2 L}\left|\lambda_{g}(\ell)\right| \ell \\
& \ll(P M L)^{\varepsilon} \frac{N L}{P M^{1 / 2}} .
\end{aligned}
$$

The last inequality is deduced by an application of Cauchy-Schwarz inequality and lemma 2.3. We similarly bound the sums in (15) corresponding to $c=1, p, M$. When $c=1$, we get arbitrarily small contribution because of the weight functions $\widetilde{W}^{ \pm}$. When $c=p$, we again get arbitrarily small contribution (because of the weight functions $\widetilde{W}^{ \pm}$) since we will choose $P$ such that

$$
P^{2}<M^{1-\delta} L
$$


When $c=M$, we have

$$
\begin{aligned}
& \frac{N^{2} g_{\chi}}{M L^{\star} P^{\star}} \sum_{ \pm} \sum_{\ell \in \mathcal{L}} \overline{\lambda_{g}(\ell)} \ell \sum_{p \in \mathcal{P}} \frac{1}{p M^{2}} \sum_{n=1}^{\infty} \lambda_{g}(n) \widetilde{W}_{v, g}^{ \pm}\left(\frac{n}{M^{2} / N \ell}\right) \\
& \times \sum_{|r| \ll M N^{\varepsilon} / N}\left(\sum_{\alpha \bmod M}^{\star} \bar{\chi}(r-\alpha \ell) e\left(\mp \frac{\bar{\alpha} n}{M}\right)\right) \widehat{V_{v}}\left(\frac{r N}{M}\right) \\
& \ll(P M L)^{\varepsilon} \frac{M}{P} .
\end{aligned}
$$

This bound is obtained by making use of Lemma A.1 to get $\sum_{\alpha \bmod M}^{\star} \bar{\chi}(r-\alpha \ell) e\left(\mp \frac{\bar{\alpha} n}{M}\right) \ll M^{1 / 2}$. Therefore from (15), (16) and (18), we obtain,

$$
S(N)=S^{\star}(N)+O\left((P M L)^{\varepsilon}\left(\frac{N L}{P M^{1 / 2}}+\frac{M}{P}+\frac{N}{L}\right)\right)
$$

under the condition $P^{2}<M^{1-\delta} L$, where

$$
\begin{aligned}
S^{\star}(N)= & \frac{N^{2} g_{\chi}}{M^{3} L^{\star} P^{\star}} \sum_{ \pm} \sum_{\ell \in \mathcal{L}} \overline{\lambda_{g}(\ell) \ell} \sum_{p \in \mathcal{P}} \frac{\chi(p)}{p^{2}} \sum_{n=1}^{\infty} \lambda_{g}(n) \widetilde{W}_{v, g}^{ \pm}\left(\frac{n}{p^{2} M^{2} / N \ell}\right) \\
& \times \sum_{|r| \ll p M N^{\varepsilon} / N}\left(\sum_{\alpha \bmod p M}^{\star} \bar{\chi}(r-\alpha \ell) e\left(\mp \frac{\bar{\alpha} n}{p M}\right) \delta(r-\alpha \ell \equiv 0 \bmod p)\right) \widehat{V_{v}}\left(\frac{r N}{p M}\right)+O\left(N^{-2018}\right) .
\end{aligned}
$$

Since $(p, M)=1$, the sum $\sum_{\alpha \bmod p M}^{\star} \bar{\chi}(r-\alpha \ell) e\left(\mp \frac{\bar{\alpha} n}{p M}\right) \delta(r-\alpha \ell \equiv 0 \bmod p)$ factors as

$$
\begin{aligned}
& \sum_{\alpha \bmod M}^{\star} \bar{\chi}(r-\alpha \ell) e\left(\mp \frac{\overline{\alpha p} n}{M}\right) \times \sum_{\alpha \bmod p}^{\star} e\left(\mp \frac{\overline{\alpha M} n}{p}\right) \delta(r-\alpha \ell \equiv 0 \bmod p) \\
& =e\left(\mp \frac{\overline{r M} n \ell}{p}\right) \sum_{\alpha \bmod M}^{\star} \bar{\chi}(r-\alpha \ell) e\left(\mp \frac{\overline{\alpha p} n}{M}\right),
\end{aligned}
$$

Since $(r, p)=1$, we have $r \neq 0$. Moreover, it suffices to estimate the 'minus' term of $S^{\star}(N)$ since the estimates of the 'plus' terms will be similar. By abuse of notation, we write $\widetilde{W}_{v, g}^{-}$as $\widetilde{W}_{v}$. Then,

$$
\begin{aligned}
S^{\star}(N)= & \frac{N^{2} g_{\chi}}{L^{\star} P^{\star} M^{3}} \sum_{n=1}^{\infty} \lambda_{g}(n) \sum_{\ell \in \mathcal{L}} \overline{\lambda_{g}(\ell)} \ell \sum_{p \in \mathcal{P}} \frac{\chi(p)}{p^{2}} \sum_{(r, p)=1} e\left(\frac{-\bar{r} n \ell \bar{M}}{p}\right) \\
& \sum_{\alpha(M)}^{\star} \bar{\chi}(r+\alpha) e\left(\frac{\bar{\alpha} n \ell \bar{p}}{M}\right) \widehat{V_{v}}\left(\frac{r}{p M / N}\right) \widetilde{W}_{v}\left(\frac{n}{p^{2} M^{2} / N \ell}\right) .
\end{aligned}
$$

Munshi treated a sum similar to ours in [18, P.13]. For the sake of completeness, we will carry out the details, but our arguments closely follow that of Munshi [18, Section 7].

Remark 5.1. (1) At this stage, if we estimate the sum $S^{\star}(N)$ directly (with the help of Lemma A.1), we would get

$$
S^{\star}(N) \ll N^{\varepsilon} \frac{N^{2} M^{1 / 2}}{L P M^{3}} \frac{P^{2} M^{2}}{N L} L^{2} \frac{1}{P} \frac{P M}{N} M^{1 / 2} \ll N^{\varepsilon} P M .
$$

This falls short of $O\left(P M^{\eta}\right)$ from the target bound $O\left(M^{1-\eta}\right)$. In the sequel, we will use Cauchy-Schwarz to smooth the $n$-variable and apply Poisson summation thereafter to obtain extra saving.

(2) It is natural to try to compare our method to that used by [5], and by [2, 8]. The ingredients are similar, the results are the same, and both methods work by embedding the modular form $g$ in question into some larger space of cusp forms on $\Gamma_{0}(M)$ and averaging, in our case indirectly via the Cauchy-Schwarz inequality, in [2, 5, 8]'s case more directly via the Petersson formula. We thank Paul Nelson for pointing out this analogy. 


\section{Cauchy-Schwarz and Poisson Summation}

In the following, whenever we need to bound the Fourier coefficients $\lambda_{g}(n)$ for $g$ a Maass form, we simply apply the Rankin-Selberg estimate $\sum_{n \leqslant X}\left|\lambda_{g}(n)\right|^{2} \ll X$, as a substitute of the Ramanujan bound for individual coefficients. Recall from the test function $\widetilde{W}_{v}$ we know that the $n$-sum is supported on $n \sim \frac{p^{2} M^{2} v^{2}}{N \ell}$. We set

$$
\mathcal{N}_{0}=\frac{p^{2} M^{2} v^{2}}{N L}
$$

Up to an arbitrarily small error, we arrive at

$$
\begin{aligned}
S^{\star}(N) \ll \frac{N^{2+\varepsilon}}{L^{\star} P^{\star} M^{5 / 2}} \sum_{n}\left|\lambda_{g}(n)\right| U\left(\frac{n}{\mathcal{N}_{0}}\right) \mid \sum_{\ell \in \mathcal{L}} \overline{\lambda_{g}(\ell) \ell} \sum_{p \in \mathcal{P}} \frac{\chi(p)}{p^{2}} \sum_{(r, p)=1} e\left(\frac{-\bar{r} n \ell \bar{M}}{p}\right) \\
\sum_{\alpha(M)}^{\star} \bar{\chi}(r+\alpha) e\left(\frac{\bar{\alpha} n \ell \bar{p}}{M}\right) \widehat{V_{v}}\left(\frac{r}{p M / N}\right) \widetilde{W}_{v}\left(\frac{n}{p^{2} M^{2} / N \ell}\right) \mid .
\end{aligned}
$$

Here $U$ is a smooth function with compact support contained in $\mathbb{R}_{>0}$. Applying the Cauchy-Schwarz inequality to the $n$-sum and using the Ramanujan bound on average,

$$
S^{\star}(N) \ll(N M L)^{\varepsilon} \frac{N^{2}}{L P M^{5 / 2}} \mathcal{N}_{0}^{1 / 2} S^{\star}\left(N, \mathcal{N}_{0}\right)^{1 / 2}+N^{-2018},
$$

where

$$
\begin{aligned}
S^{\star}\left(N, \mathcal{N}_{0}\right)=\sum_{n} U\left(\frac{n}{\mathcal{N}_{0}}\right) \mid \sum_{\ell \in \mathcal{L}} \overline{\lambda_{g}(\ell)} \ell & \sum_{p \in \mathcal{P}} \frac{\chi(p)}{p^{2}} \sum_{(r, p)=1} e\left(\frac{-\bar{r} n \ell \bar{M}}{p}\right) \\
& \times\left.\sum_{\alpha(M)}^{\star} \bar{\chi}(r+\alpha) e\left(\frac{\bar{\alpha} n \ell \bar{p}}{M}\right) \widehat{V_{v}}\left(\frac{r}{p M / N}\right) \widetilde{W}_{v}\left(\frac{n}{p^{2} M^{2} / N \ell}\right)\right|^{2} .
\end{aligned}
$$

Opening the square above and switching the order of summations, it suffices to bound the following

$$
\begin{aligned}
S^{\star}\left(N, \mathcal{N}_{0}\right)= & \sum_{\ell_{1} \in \mathcal{L}} \overline{\lambda_{g}\left(\ell_{1}\right)} \ell_{1} \sum_{\ell_{2} \in \mathcal{L}} \lambda_{g}\left(\ell_{2}\right) \ell_{2} \sum_{p_{1} \in \mathcal{P}} \sum_{p_{2} \in \mathcal{P}} \frac{\chi\left(p_{1}\right) \bar{\chi}\left(p_{2}\right)}{\left(p_{1} p_{2}\right)^{2}} \\
& \sum_{\left(r_{1}, p_{1}\right)=1} \sum_{\left(r_{2}, p_{2}\right)=1} \widehat{V_{v}}\left(\frac{r_{1}}{p_{1} M / N}\right) \frac{\widehat{V}_{v}\left(\frac{r_{2}}{p_{2} M / N}\right)}{\sum_{\alpha_{1}(M)}} \sum^{\star} \bar{\chi}\left(r_{1}+\alpha_{1}\right) \sum_{\alpha_{2}(M)}^{\star} \chi\left(r_{2}+\alpha_{2}\right) \times \mathbf{T},
\end{aligned}
$$

with

$$
\begin{aligned}
\mathbf{T}=\sum_{n=1}^{\infty} e\left(\frac{-\overline{r_{1}} n \ell_{1} \bar{M}}{p_{1}}\right) & e\left(\frac{\overline{r_{2}} n \ell_{2} \bar{M}}{p_{2}}\right) e\left(\frac{\overline{\alpha_{1}} n \ell_{1} \overline{p_{1}}-\overline{\alpha_{2}} n \ell_{2} \overline{p_{2}}}{M}\right) \\
& \times U\left(\frac{n}{\mathcal{N}_{0}}\right) \widetilde{W}_{v}\left(\frac{n}{p_{1}^{2} M^{2} / N \ell_{1}}\right) \overline{W_{v}}\left(\frac{n}{p_{2}^{2} M^{2} / N \ell_{2}}\right) .
\end{aligned}
$$

We note that one can truncate the $r_{1}, r_{2}$-sums in (21) at $\left|r_{1}\right|,\left|r_{2}\right| \ll N^{\varepsilon} \frac{P M}{N}$, at the cost of a negligible error. For smaller values of $r_{1}$ and $r_{2}$, we will use the trivial bounds $\widehat{V_{v}}\left(\frac{r_{1}}{p_{1} M / N}\right), \widehat{V_{v}}\left(\frac{r_{2}}{p_{2} M / N}\right) \ll 1$.

Breaking the above $n$-sum modulo $p_{1} p_{2} M$ and applying Poisson summation to it,

$$
\begin{aligned}
\mathbf{T}= & \frac{\mathcal{N}_{0}}{p_{1} p_{2} M} \sum_{n} \sum_{b \bmod p_{1} p_{2} M} e\left(\frac{-\overline{r_{1}} b \ell_{1} \bar{M}}{p_{1}}\right) e\left(\frac{\overline{r_{2}} b \ell_{2} \bar{M}}{p_{2}}\right) \\
& e\left(\frac{\overline{\alpha_{1}} b \ell_{1} \overline{p_{1}}-\overline{\alpha_{2}} b \ell_{2} \overline{p_{2}}}{M}\right) e\left(\frac{b n}{p_{1} p_{2} M}\right) \mathcal{J}\left(\frac{n}{p_{1} p_{2} M / \mathcal{N}_{0}}\right),
\end{aligned}
$$

where

$$
\mathcal{J}\left(\frac{n}{p_{1} p_{2} M / \mathcal{N}_{0}}\right):=\int_{\mathbb{R}} U(x) e\left(-\frac{n \mathcal{N}_{0} x}{p_{1} p_{2} M}\right) \widetilde{W}_{v}\left(\frac{x \mathcal{N}_{0}}{p_{1}^{2} M^{2} / N \ell_{1}}\right) \overline{\widetilde{W}_{v}\left(\frac{x \mathcal{N}_{0}}{p_{2}^{2} M^{2} / N \ell_{2}}\right)} \mathrm{d} x .
$$


The integral $\mathcal{J}\left(\frac{n}{p_{1} p_{2} M / \mathcal{N}_{0}}\right)$ gives arbitrarily power saving in $N$ if $|n| \gg N^{\varepsilon} \frac{p_{1} p_{2} M}{\mathcal{N}_{0}}$. Hence we can truncate the dual $n$-sum at

$$
|n| \ll N^{\varepsilon} \frac{N L}{M v^{2}}
$$

at the cost of a negligible error. For smaller values of $n$, we use the trivial bound $\mathcal{J}\left(\frac{n}{p_{1} p_{2} M / \mathcal{N}_{0}}\right) \ll 1$. Since $\left(p_{1} p_{2}, M\right)=1$, we apply reciprocity to write

$$
\begin{aligned}
\mathbf{T}= & \frac{\mathcal{N}_{0}}{p_{1} p_{2} M} \sum_{n} \sum_{b \bmod p_{1} p_{2}} e\left(\frac{\left(-\overline{r_{1}} \ell_{1} p_{2}+\overline{r_{2}} \ell_{2} p_{1}+n\right) \bar{M} b}{p_{1} p_{2}}\right) \\
& \times \sum_{b \bmod M} e\left(\frac{\left(\overline{\alpha_{1}} \ell_{1} p_{2}-\overline{\alpha_{2}} \ell_{2} p_{1}+n\right) \overline{p_{1} p_{2}} b}{M}\right) \mathcal{J}\left(\frac{n}{p_{1} p_{2} M / \mathcal{N}_{0}}\right) \\
= & \mathcal{N}_{0} \sum_{\substack{|n| \ll N^{\varepsilon} \frac{p_{1} p_{2} M}{\mathcal{N}_{0}} \\
-\overline{r_{1}} \ell_{1} p_{2}+\overline{r_{2}} \ell_{2} p_{1}+n \equiv \bmod p_{1} p_{2} \\
\left(\overline{\alpha_{1}} \ell_{1} p_{2}+n, M\right)=1}} \delta\left(\alpha_{2} \equiv \ell_{2} p_{1}\left(\overline{\overline{\alpha_{1}} \ell_{1} p_{2}+n}\right) \bmod M\right) \mathcal{J}\left(\frac{n}{p_{1} p_{2} M / \mathcal{N}_{0}}\right)+O\left(N^{-20180}\right) .
\end{aligned}
$$

We must clarify that $\overline{r_{i}}$ is the inverse of $r_{i} \bmod p_{i}\left(\right.$ and not $\left.\bmod p_{1} p_{2}\right)$. Substituting the above into (21),

$$
\begin{aligned}
& S^{\star}\left(N, \mathcal{N}_{0}\right) \ll \mathcal{N}_{0} \sum_{\ell_{1} \in \mathcal{L}}\left|\overline{\lambda_{g}\left(\ell_{1}\right)} \ell_{1}\right| \sum_{\ell_{2} \in \mathcal{L}}\left|\lambda_{g}\left(\ell_{2}\right) \ell_{2}\right| \sum_{p_{1} \in \mathcal{P}} \sum_{p_{2} \in \mathcal{P}} \frac{1}{\left(p_{1} p_{2}\right)^{2}} \\
& \sum_{\substack{0 \neq\left|r_{1}\right| \ll R \\
\left(r_{1}, p_{1}\right)=1}} \sum_{\substack{0 \neq\left|r_{2}\right| \ll R \\
\left(r_{2}, p_{2}\right)=1}} \sum_{\substack{|n| \ll \bar{r}_{1} \ell_{1} p_{2}+\overline{r_{2}} \ell_{2} p_{1}+p_{1} p_{2} M \\
\mathcal{N}_{0}}}|\mathfrak{C}|+N^{-2018},
\end{aligned}
$$

where

$$
\mathfrak{C}=\sum_{\substack{\alpha \bmod M \\\left(\bar{\alpha} \ell_{1} p_{2}+n, M\right)=1}}^{\star} \bar{\chi}\left(r_{1}+\alpha\right) \chi\left(r_{2}+\ell_{2} p_{1}\left(\overline{\bar{\alpha} \ell_{1} p_{2}+n}\right)\right)
$$

and

$$
R:=N^{\varepsilon} P M / N
$$

When $\ell_{1} \neq \ell_{2}$, we apply the Cauchy-Schwarz inequality to the $\ell_{i}$-sums to get rid of the Fourier coefficients $\lambda_{g}\left(\ell_{i}\right)$ by using the Ramanujan bound on average. Then,

$$
S^{\star}\left(N, \mathcal{N}_{0}\right) \ll S_{0}^{\star}\left(N, \mathcal{N}_{0}\right)+S_{1}^{\star}\left(N, \mathcal{N}_{0}\right)+N^{-2018}
$$

where

$$
S_{0}^{\star}\left(N, \mathcal{N}_{0}\right)=\mathcal{N}_{0} \sum_{\ell \in \mathcal{L}}\left|\lambda_{g}(\ell)\right|^{2} \ell^{2} \sum_{p_{1} \in \mathcal{P}} \sum_{p_{2} \in \mathcal{P}} \frac{1}{\left(p_{1} p_{2}\right)^{2}} \sum_{\substack{0 \neq\left|r_{1}\right| \ll R \\\left(r_{1}, p_{1}\right)=1}} \sum_{\substack{0 \neq\left|r_{2}\right| \ll R \\\left(r_{2}, p_{2}\right)=1}} \sum_{\substack{|n| \ll N_{1} \ell \frac{p_{1} p_{2} M}{\mathcal{N}_{0}} \\\left(r_{2}+\overline{r_{2}} \ell p_{1}+n \equiv 0 \bmod p_{1} p_{2}\right.}}|\mathfrak{C}|,
$$

and

$$
\begin{aligned}
& S_{1}^{\star}\left(N, \mathcal{N}_{0}\right)=\mathcal{N}_{0} L^{1+\varepsilon}\left(\sum _ { \substack { \ell _ { 1 } \in \mathcal { L } \\
\ell _ { 1 } \neq \ell _ { 2 } } } \sum _ { \ell _ { 2 } \in \mathcal { L } } \ell _ { 1 } ^ { 2 } \ell _ { 2 } ^ { 2 } \left[\sum_{p_{1} \in \mathcal{P}} \sum_{p_{2} \in \mathcal{P}} \frac{1}{\left(p_{1} p_{2}\right)^{2}}\right.\right. \\
& \left.\left.\times \sum_{\substack{0 \neq\left|r_{1}\right| \ll R \\
\left(r_{1}, p_{1}\right)=1}} \sum_{\substack{0 \neq\left|r_{2}\right| \ll R \\
\left(r_{2}, p_{2}\right)=1}} \sum_{\substack{|n| \ll N_{1} \ell_{1} p_{2}+\bar{r}_{2} \ell_{2} p_{1}+n \equiv 0 \bmod p_{1} p_{2} \\
\mathcal{N}_{0}}}|\mathfrak{C}|\right]^{2}\right)^{1 / 2} .
\end{aligned}
$$

We will choose $P<M^{1-\delta-\varepsilon}$, so that $R<M$ and therefore $\left(r_{1} r_{2}, M\right)=1$. The remaining task is to count the number of points satisfying the congruence conditions and bounding the sums.

We divide our analysis into cases and write $S_{0}^{\star}\left(N, \mathcal{N}_{0}\right) \ll \mathcal{N}_{0}\left(\Delta_{1}+\Delta_{2}\right)$ and $S_{1}^{\star}\left(N, \mathcal{N}_{0}\right) \ll \mathcal{N}_{0} L^{\varepsilon}\left(\Sigma_{1}+\Sigma_{2}\right)^{1 / 2}$. The contribution of the terms with $n \equiv 0 \bmod M$ is given by $\Delta_{1}$ and $\Sigma_{1}$, and the contribution of the terms with $n \not \equiv 0 \bmod M$ is given by $\Delta_{2}$ and $\Sigma_{2}$, with $\Delta_{i}$ and $\Sigma_{j}$ appropriately defined. 
6.1. $\underline{n \equiv 0 \bmod M}$. For the sum (23), Lemma A.2 (with the parameter $(\alpha, \beta)=\left(\ell_{2} p_{1}, \ell_{1} p_{2}\right)$ being applied) shows that

$$
\mathfrak{C}=\chi\left(\ell_{2} p_{1} \overline{\ell_{2} p_{1}}\right) R_{M}\left(r_{2}-r_{1} \ell_{2} p_{1} \overline{\ell_{1} p_{2}}\right)-\chi\left(r_{2} \overline{r_{1}}\right)= \begin{cases}O(M), & \text { if } r_{2} \ell_{1} p_{2} \equiv r_{1} \ell_{2} p_{1} \bmod M \\ O(1), & \text { otherwise. }\end{cases}
$$

According to $r_{2} \ell_{1} p_{2} \equiv r_{1} \ell_{2} p_{1} \bmod M$ or not, we write

$$
\Delta_{1}=\Delta_{10}+\Delta_{11} \quad \text { and } \quad \Sigma_{1}=\Sigma_{10}+\Sigma_{11}
$$

where

$$
\Delta_{10}:=\sum_{\ell \in \mathcal{L}}\left|\lambda_{g}(\ell)\right|^{2} \ell^{2} \sum_{p_{1} \in \mathcal{P}} \sum_{p_{2} \in \mathcal{P}} \frac{1}{\left(p_{1} p_{2}\right)^{2}} \sum_{\substack{0 \neq\left|r_{1}\right| \ll R \\\left(r_{1}, p_{1}\right)=1 \\ r_{2} p_{2} \equiv r_{1} p_{1} \bmod M}} \sum_{\substack{0 \neq\left|r_{2}\right| \ll R \\\left(r_{2}, p_{2}\right)=1}} \sum_{\substack{|n| \bar{r}_{1} \ell p_{2}+p_{2} \ell N_{1} \frac{p_{1} p_{2} M}{N_{0}} \\ n \equiv 0 \bmod M}} M,
$$

and

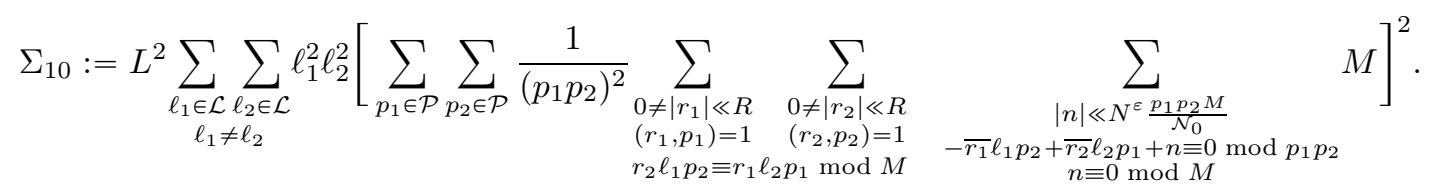

$\Delta_{11}$ and $\Sigma_{11}$ are the other pieces with the congruence condition $r_{2} \ell_{1} p_{2} \not \equiv r_{1} \ell_{2} p_{1} \bmod M$. Opening the square, we write $\Sigma_{10}$ and $\Sigma_{11}$ as a sum over $\ell_{i}, n, n^{\prime}, r_{i}, r_{i}^{\prime}, p_{i}, p_{i}^{\prime}$ for $i=1,2$. Then, under the assumption $P^{2} L<N^{1-\varepsilon}$, Lemmas A.3 and A.4 give

$$
\begin{array}{ccc}
\Sigma_{10} \ll(P M L)^{\varepsilon} \frac{L^{6} M^{4}}{P^{4} N^{2}} & \text { and } & \Sigma_{11} \ll(P M L)^{\varepsilon} \frac{L^{8} M^{4}}{N^{4} P^{4}}\left(1+\frac{P^{2}}{\mathcal{N}_{0}}\right)^{2}, \\
\Delta_{10} \ll(P M L)^{\varepsilon} \frac{L^{3} M^{2}}{P^{2} N} & \text { and } & \Delta_{11} \ll(P M L)^{\varepsilon} \frac{L^{3} M^{2}}{N^{2} P^{2}}\left(1+\frac{P^{2}}{\mathcal{N}_{0}}\right) .
\end{array}
$$

6.2. $n \not \equiv 0 \bmod M$. Lemma A.2 shows that

$$
\mathfrak{C}= \begin{cases}O(M), & \text { if } r_{1}-\bar{n} \ell_{1} p_{2} \equiv r_{2}+\bar{n} \ell_{2} p_{1} \equiv 0 \bmod M \\ O\left(M^{1 / 2}\right), & \text { otherwise. }\end{cases}
$$

According to $r_{1}-\bar{n} \ell_{1} p_{2} \equiv r_{2}+\bar{n} \ell_{2} p_{1} \equiv 0 \bmod M$ or not, we write

$$
\Delta_{2}=\Delta_{20}+\Delta_{21} \quad \text { and } \quad \Sigma_{2}=\Sigma_{20}+\Sigma_{21},
$$

where

$$
\Delta_{20}:=\sum_{\ell \in \mathcal{L}}\left|\lambda_{g}(\ell)\right|^{2} \ell^{2} \sum_{p_{1} \in \mathcal{P}} \sum_{p_{2} \in \mathcal{P}} \frac{1}{\left(p_{1} p_{2}\right)^{2}} \sum_{\substack{0 \neq\left|r_{1}\right| \ll R \\\left(r_{1}, p_{1}\right)=1}} \sum_{\substack{0 \neq\left|r_{2}\right| \ll R \\\left(r_{2}, p_{2}\right)=1}} \sum_{\substack{|n| \ll N^{\varepsilon} \frac{p_{1} p_{2} M}{\mathcal{N}} \\-\bar{r}_{1} \ell p_{2}+\overline{r_{2}} p_{1}+n=0 \bmod p_{1} p_{2} \\ n \neq 0 \bmod M \\ r_{1}-\bar{n} \ell p_{2} \equiv r_{2}+\bar{n} \ell p_{1} \equiv 0 \bmod M}} M,
$$

and

$$
\Sigma_{20}:=L^{2} \sum_{\substack{\ell_{1} \in \mathcal{L} \\
\ell_{1} \neq \ell_{2} \in \mathcal{L}}} \sum_{1}^{2} \ell_{2}^{2}\left[\sum_{p_{1} \in \mathcal{P}} \sum_{p_{2} \in \mathcal{P}} \frac{1}{\left(p_{1} p_{2}\right)^{2}} \sum_{\begin{array}{c}
0 \neq\left|r_{1}\right| \ll R \\
\left(r_{1}, p_{1}\right)=1
\end{array}} \sum_{\substack{0 \neq\left|r_{2}\right| \ll R \\
\left(r_{2}, p_{2}\right)=1}} \sum_{\substack{|n| \ll N^{\varepsilon} \frac{p_{1} p_{2} M}{\mathcal{N}_{0}} \\
-\overline{r_{1}} \ell_{1} p_{2}+\overline{r_{2}} \ell_{2} p_{1}+n=0 \bmod p_{1} p_{2} \\
n \neq 0 \bmod M \\
r_{1}-\bar{n} \ell_{1} p_{2} \equiv r_{2}+\bar{n} \ell_{2} p_{1} \equiv 0 \bmod M}} M\right]^{2} .
$$

$\Delta_{21}$ and $\Sigma_{21}$ are the other pieces. Opening the square, we write $\Sigma_{20}$ and $\Sigma_{21}$ as a sum over $\ell_{i}, n, n^{\prime}, r_{i}, r_{i}^{\prime}, p_{i}, p_{i}^{\prime}$ for $i=1,2$. Then, from Lemmas A.5 and A.6

$$
\begin{aligned}
& \Sigma_{20} \ll(P M L)^{\varepsilon} \frac{L^{6} M^{4}}{P^{4} N^{2}} \quad \text { and } \quad \Sigma_{21} \ll(P M L)^{\varepsilon} \frac{L^{8} M^{5}}{N^{4} P^{4}}\left(1+\frac{P^{2} M}{\mathcal{N}_{0}}\right)^{2}, \\
& \Delta_{20} \ll(P M L)^{\varepsilon} \frac{L^{3} M^{2}}{P^{2} N} \quad \text { and } \quad \Delta_{21} \ll(P M L)^{\varepsilon} \frac{L^{3} M^{5 / 2}}{N^{2} P^{2}}\left(1+\frac{P^{2} M}{\mathcal{N}_{0}}\right) \text {. }
\end{aligned}
$$


6.3. Conclusion. The bounds (24) and (25) imply

$$
S^{\star}\left(N, \mathcal{N}_{0}\right) \ll(P M L)^{\varepsilon} \mathcal{N}_{0}\left[\frac{L^{3} M^{2}}{P^{2} N}+\frac{L^{4} M^{5 / 2}}{N^{2} P^{2}}\left(1+\frac{P^{2} M}{\mathcal{N}_{0}}\right)\right] .
$$

Inserting this into (20),

$$
S^{\star}(N) \ll(P M L)^{\varepsilon}\left[\frac{N^{1 / 2} M^{1 / 2} v^{2}}{L^{1 / 2}}+M^{3 / 4}+N^{1 / 2} L^{1 / 2} M^{1 / 4} v\right] .
$$

Recalling that $v=M^{\varepsilon}(12)$, and inserting the above into (19),

$$
\frac{S(N)}{N^{1 / 2}} \ll(P M L)^{\varepsilon}\left[\frac{N^{1 / 2} L}{P M^{1 / 2}}+\frac{M}{P N^{1 / 2}}+\frac{M^{1 / 2}}{L^{1 / 2}}+\frac{M^{3 / 4}}{N^{1 / 2}}+L^{1 / 2} M^{1 / 4}\right] .
$$

The first term is small. Comparing the rest of the terms and the trivial bound of $N^{1 / 2}$, the optimal choices of parameters turn out to be $P=M^{1 / 4+\varepsilon}$ and $L=P^{1-\varepsilon}$. Therefore the conditions in (10), (17) and (27) are satisfied. In that case,

$$
\frac{S(N)}{N^{1 / 2}} \ll M^{\varepsilon}\left(\frac{M^{3 / 4}}{N^{1 / 2}}+M^{3 / 8}\right) .
$$

It therefore makes sense to take $N>M^{3 / 4}$. For $N \ll M^{3 / 4}$, we use the trivial bound of $N^{1 / 2}$. We obtain

$$
L\left(\frac{1}{2}, g \otimes \chi\right) \ll M^{3 / 8+\varepsilon} .
$$

That proves Theorem 1.1 and Theorem 1.2.

\section{Appendix A. Shifted Character sums And COUnting Lemmas}

For this section, let $M>3$ be a prime and define

$$
\mathfrak{K}=\sum_{z \in \mathbb{F}_{M}^{\times}} \bar{\chi}(r+\ell z) e\left(\frac{n \bar{z}}{M}\right), \quad(\ell, M)=1, \quad n, r, \ell \in \mathbb{Z} .
$$

Lemma A.1. Suppose that $(r, M)=1$. If $M \mid n$, then $\mathfrak{K}=-\bar{\chi}(r)$. If $M \nmid n$, then

$$
\mathfrak{K} \ll M^{1 / 2} .
$$

Proof. For $M \mid n$, trivial. If $M \nmid n$, by the Fourier expansion of $\chi$ in terms of additive characters

$$
\chi(a)=g_{\bar{\chi}}^{-1} \sum_{y \bmod M} \bar{\chi}(y) e\left(\frac{a y}{M}\right)
$$

we have

$$
\mathfrak{K}=g_{\chi}^{-1} \sum_{y, z \in \mathbb{F}_{M}^{\times}} \chi(y) e\left(\frac{r y+\ell y z+n \bar{z}}{M}\right) .
$$

Then the bound follows from [1, Corollary 4.3].

We define

$$
\mathfrak{C}=\sum_{\substack{z \in \mathbb{F}_{M}^{\times} \\(n+\beta \bar{z}, M)=1}} \bar{\chi}\left(r_{1}+z\right) \chi\left(r_{2}+\alpha(\overline{n+\beta \bar{z}})\right), \quad(\alpha \beta, M)=1, \quad n, r_{1}, r_{2}, \alpha, \beta \in \mathbb{Z} .
$$

Lemma A.2. Suppose that $\left(r_{1} r_{2}, M\right)=1$. If $M \mid n$, we have

$$
\mathfrak{C}=\chi(\alpha \bar{\beta}) R_{M}\left(r_{2}-r_{1} \alpha \bar{\beta}\right)-\chi\left(r_{2} \overline{r_{1}}\right),
$$

where $R_{M}(a)=\sum_{z \in \mathbb{F}_{M}^{\times}} e(a z / M)$ is the Ramanujan sum. If $M \nmid n$ and at least one of $r_{1}-\bar{n} \beta$ and $r_{2}+\bar{n} \alpha$ is nonzero in $\mathbb{F}_{M}$, then

$$
\mathfrak{C} \ll M^{1 / 2}
$$


Finally, if $n \neq 0$ and $r_{1}-\bar{n} \beta=r_{2}+\bar{n} \alpha=0$ in $\mathbb{F}_{M}$, then

$$
\mathfrak{C}= \begin{cases}-\chi\left(n r_{2} \bar{\beta}\right) & \text { if } \chi \text { is not a quadratic character }, \\ \chi\left(\bar{n} r_{2} \beta\right)(M-1) & \text { if } \chi \text { is a quadratic character. }\end{cases}
$$

Proof. For $M \mid n$,

$$
\mathfrak{C}=\sum_{z \in \mathbb{F}_{M}} \bar{\chi}\left(r_{1}+z\right) \chi\left(r_{2}+\alpha \bar{\beta} z\right)-\chi\left(r_{2} \overline{r_{1}}\right) .
$$

Then the first statement follows from (26). If $M \nmid n$, by making change of variables $z \rightarrow \bar{n} \beta z$ and $z+1 \rightarrow z$, we have

$$
\mathfrak{C}=\sum_{z \in \mathbb{F}_{M}^{\times}} \bar{\chi}\left(r_{1}+\bar{n} \beta(z-1)\right) \chi\left(r_{2}+\bar{n} \alpha(1-\bar{z})\right)-\chi\left(r_{2} \overline{r_{1}}\right) .
$$

Applying (26) again we get

$$
\mathfrak{C}=g_{\chi}^{-1} g_{\bar{\chi}}^{-1} \sum_{x, y, z \in \mathbb{F}_{M}^{\times}} \chi(x) \bar{\chi}(y) e\left(\frac{\left(r_{1}-\bar{n} \beta\right) x+\left(r_{2}+\bar{n} \alpha\right) y+\bar{n} \beta x z-\bar{n} \alpha y \bar{z}}{M}\right)-\chi\left(r_{2} \overline{r_{1}}\right) .
$$

Consider the Newton polyhedron $\Delta(f)$ of

$$
f(x, y, z)=\left(r_{1}-\bar{n} \beta\right) x+\left(r_{2}+\bar{n} \alpha\right) y+\bar{n} \beta x z-\bar{n} \alpha y z^{-1} \in \mathbb{F}_{M}^{\times}\left[x, y, z,(x y z)^{-1}\right] .
$$

We separate into two cases.

(1) If $r_{1}-\bar{n} \beta=0$ or $r_{2}+\bar{n} \alpha=0$ in $\mathbb{F}_{M}$, then $\Delta(f)$ is the tetrahedron in $\mathbb{R}^{3}$ with vertices $(0,0,0),(0,1,0)$, $(1,0,1),(0,1,-1)$ or $(0,0,0),(1,0,0),(1,0,1),(0,1,-1)$. It is easy to check that $f$ is nondegenerate with respect to $\Delta(f)$. By [1] (see also [9]), we have $\mathfrak{C} \ll M^{1 / 2}$.

(2) If both $r_{1}-\bar{n} \beta$ and $r_{2}+\bar{n} \alpha$ are nonzero in $\mathbb{F}_{M}$, then $\Delta(f)$ is the pentahedron in $\mathbb{R}^{3}$ with vertices $(0,0,0),(1,0,0),(0,1,0),(1,0,1)$ and $(0,1,-1)$. The only face which fails to meet the criterion for nondegeneracy is the quadrilateral one with polynomial $f_{\sigma}(x, y, z)=\left(r_{1}-\bar{n} \beta\right) x+\left(r_{2}+\bar{n} \alpha\right) y+$ $\bar{n} \beta x z-\bar{n} \alpha y z^{-1}$ for which the locus of $\partial f_{\sigma} / \partial x=\partial f_{\sigma} / \partial y=\partial f_{\sigma} / \partial z=0$ is empty in $\left(\mathbb{F}_{M}^{\times}\right)^{3}$ only if $n \not \equiv \beta \overline{r_{1}}-\alpha \overline{r_{2}} \bmod M$. Therefore, for $n \not \equiv \beta \overline{r_{1}}-\alpha \overline{r_{2}} \bmod M$, we can apply the square-root cancellation result in [1] or [9] to get $\mathfrak{C} \ll M^{1 / 2}$.

For $n \equiv \beta \overline{r_{1}}-\alpha \overline{r_{2}} \bmod M$, we have $r_{1}-\bar{n} \beta \equiv-\alpha r_{1} \overline{n r_{2}} \bmod M$ and $r_{2}+\bar{n} \alpha \equiv \beta r_{2} \overline{n r_{1}} \bmod M$. By changing variables $x \rightarrow x \bar{z}$ and $\bar{n} x \rightarrow x, \bar{n} y \rightarrow y$ we obtain

$$
\mathfrak{C}=g_{\chi}^{-1} g_{\bar{\chi}}^{-1} \sum_{x, y, z \in \mathbb{F}_{M}^{\times}} \chi(x) \bar{\chi}(y) \bar{\chi}(z) e\left(\frac{\left(r_{1} \overline{r_{2}} x+y\right)\left(\beta r_{2} \overline{r_{1}}-\alpha \bar{z}\right)}{M}\right)-\chi\left(r_{2} \overline{r_{1}}\right) .
$$

Since $\chi$ is primitive, the sums over $x$ and $y$ vanishes if $z \equiv \alpha r_{1} \overline{\beta r_{2}} \bmod M$. Thus we can make change of variables $\left(\beta r_{2} \overline{r_{1}}-\alpha \bar{z}\right) x \rightarrow x$ and $\left(\beta r_{2} \overline{r_{1}}-\alpha \bar{z}\right) y \rightarrow y$ to get

$$
\begin{aligned}
\mathfrak{C} & =g_{\chi}^{-1} g_{\bar{\chi}}^{-1} \sum_{\substack{z \in \mathbb{F}_{M}^{\times} \\
z \neq \alpha r_{1} \overline{\beta r_{2}} \bmod M}} \bar{\chi}(z) \sum_{x, y \in \mathbb{F}_{M}^{\times}} \chi(x) \bar{\chi}(y) e\left(\frac{r_{1} \overline{r_{2}} x+y}{M}\right)-\chi\left(r_{2} \overline{r_{1}}\right) \\
& =-\chi^{2}\left(r_{2} \overline{r_{1}}\right) \chi(\beta \bar{\alpha})-\chi\left(r_{2} \overline{r_{1}}\right) .
\end{aligned}
$$

Finally, if $n \neq 0$ and $r_{1}-\bar{n} \beta=r_{2}+\bar{n} \alpha=0$ in $\mathbb{F}_{M}$,

$$
\begin{aligned}
\mathfrak{C} & =\chi(n) \sum_{z \in \mathbb{F}_{M}^{\times}} \bar{\chi}\left(r_{1}+z\right) \bar{\chi}(n+\beta \bar{z}) \chi\left(r_{2}+\bar{n} \alpha+r_{2} \beta \overline{z n}\right) \\
& =\chi\left(r_{2} \beta\right) \sum_{z \in \mathbb{F}_{M}^{\times}} \bar{\chi}\left(r_{1}+z\right) \bar{\chi}(n z+\beta)=\chi\left(r_{2} \beta\right) \sum_{\substack{z \in \mathbb{F}_{M} \\
z \neq \bar{n} \beta}} \bar{\chi}\left(r_{1}-\bar{n} \beta+z\right) \bar{\chi}(n z) \\
& =\chi\left(\bar{n} r_{2} \beta\right) \sum_{\substack{z \in \mathbb{F}_{M} \\
z \neq \bar{n} \beta}} \bar{\chi}^{2}(z) .
\end{aligned}
$$


If $\chi$ is not a quadratic character, then by orthogonality of characters, $\mathfrak{C}=-\chi\left(n r_{2} \bar{\beta}\right)$. If $\chi$ is a quadratic character, then $\mathfrak{C}=\chi\left(\bar{n} r_{2} \beta\right)(M-1)$.

Lemma A.3. $\Sigma_{10} \ll(P M L)^{\varepsilon} \frac{L^{6} M^{4}}{P^{4} N^{2}}$ and $\Delta_{10} \ll(P M L)^{\varepsilon} \frac{L^{3} M^{2}}{P^{2} N}$.

Proof. We recall $\left|r_{2}\right|<R:=N^{\varepsilon} P M / N$. Suppose

$$
P^{2} L \ll N^{1-\varepsilon} .
$$

Then the congruence $r_{2} \ell_{1} p_{2} \equiv r_{1} \ell_{2} p_{1} \bmod M$ implies that $r_{2} \ell_{1} p_{2}=r_{1} \ell_{2} p_{1}$. Similarly, $r_{2}^{\prime} \ell_{1} p_{2}^{\prime}=r_{1}^{\prime} \ell_{2} p_{1}^{\prime}$. Therefore fixing $\ell_{1}, p_{2}, r_{2}$ fixes $\ell_{2}, p_{1}, r_{1}$ up to factors of $\log M$. If $\ell_{1} \neq \ell_{2}$, then the equality $r_{2}^{\prime} \ell_{1} p_{2}^{\prime}=r_{1}^{\prime} \ell_{2} p_{1}^{\prime}$ implies $\ell_{2} \mid r_{2}^{\prime}$. That saves a factor of $L$ in $r_{2}^{\prime}$ sum. Further, for fixed $p_{2}^{\prime}, r_{2}^{\prime}$, there are only $\log M$ many $p_{1}^{\prime}, r_{1}^{\prime}$. In the case $\ell_{1}=\ell_{2}$, the previous identities become $r_{2} p_{2}=r_{1} p_{1}$. Therefore fixing $r_{2}, p_{2}$ fixes $r_{1}, p_{1}$ up to factors of $\log M$.

Finally, the congruence conditions on $r_{1}, r_{2}$ and $n$ can be combined to write

$$
-\overline{r_{1}} \ell_{1} p_{2}+\bar{r}_{2} \ell_{2} p_{1}+n \equiv 0 \bmod p_{1} p_{2} M \text {. }
$$

From (22), the $n$ satisfies $|n| \ll N^{1+\varepsilon} L / M$, which is smaller than the size of the modulus $p_{1} p_{2} M$. Therefore for fixed $r_{i}, \ell_{i}, p_{i}$, the $n$ sum is at most singleton. Similarly, $n^{\prime}$ is at most a singleton. Therefore up to a factor of $(P M L)^{\varepsilon}$,

$$
\Sigma_{10} \ll L^{5} L^{2} \frac{1}{P^{3}} R \frac{1}{P^{3}} \frac{R}{L} M^{2} \ll \frac{L^{6} M^{4}}{P^{4} N^{2}}, \quad \text { and } \quad \Delta_{10} \ll L^{3} \frac{1}{P^{3}} R M \ll \frac{L^{3} M^{2}}{P^{2} N} .
$$

Lemma A.4. $\Sigma_{11} \ll(P M L)^{\varepsilon} \frac{L^{8} M^{4}}{N^{4} P^{4}}\left(1+\frac{P^{2}}{\mathcal{N}_{0}}\right)^{2}$ and $\Delta_{11} \ll(P M L)^{\varepsilon} \frac{L^{3} M^{2}}{N^{2} P^{2}}\left(1+\frac{P^{2}}{\mathcal{N}_{0}}\right)$.

Proof. We let the variables of summation $\ell_{i}, p_{i}, r_{i}, n$ to be the same as before. The expressions for $\Delta_{11}$ and $\Sigma_{11}$ are the same as $\Delta_{10}$ and $\Sigma_{10}$ with the condition $r_{2} \ell_{1} p_{2} \equiv r_{1} \ell_{2} p_{1} \bmod M$ replaced by $r_{2} \ell_{1} p_{2} \not \equiv r_{1} \ell_{2} p_{1} \bmod M$. First let $\ell_{1} \neq \ell_{2}$. If $p_{1} \neq p_{2}$, then $\left(n, p_{1} p_{2}\right)=1, r_{1} \equiv \overline{n M} \ell_{1} p_{2} \bmod p_{1}$ and $r_{2} \equiv-\overline{n M} \ell_{2} p_{1} \bmod p_{2}$. Since $R \gg P$, these congruence conditions therefore save a factor of $O(P)$ in each $r_{i}$-sum. Similarly we save a factor of $O(P)$ in each $r_{i}^{\prime}$ sum. The congruence $n \equiv 0 \bmod M\left(\operatorname{resp} n^{\prime} \equiv 0 \bmod M\right)$ saves a factor of at most $M$ in the $n$-sum (resp $n^{\prime}$-sum). If $p_{1}=p_{2}=p$, then the congruence conditions imply $p \mid n$. We already have $M \mid n$. Recall from (22) the $n$-sum satisfies $|n| \ll N^{1+\varepsilon} L / M$, which is smaller than $p M$ by our choice of $P$ and $L$. Hence we have $n=0$. The remaining congruence condition $r_{1} \ell_{2} \equiv r_{2} \ell_{1} \bmod p$ shows that fixing $r_{1}, \ell_{2}, \ell_{1}$ saves a factor of $O(P)$ in the $r_{2}$-sum. The exact same savings follow for $n^{\prime}$ and $r_{2}^{\prime}$ sums. Also, the exact same analysis as done for $p_{i}, r_{i}, n$-sums follows for the case $\ell_{1}=\ell_{2}$. Therefore up to a factor of $(P M L)^{\varepsilon}$,

$$
\begin{aligned}
& \Sigma_{11} \ll L^{8}\left[\frac{1}{P^{2}} \frac{R^{2}}{P^{2}}\left(1+\frac{P^{2}}{\mathcal{N}_{0}}\right)\right]^{2}+L^{8}\left[\frac{1}{P^{3}} \frac{R^{2}}{P}\right]^{2} \ll \frac{L^{8} M^{4}}{N^{4} P^{4}}\left(1+\frac{P^{2}}{\mathcal{N}_{0}}\right)^{2}, \\
& \Delta_{11} \ll L^{3}\left[\frac{1}{P^{2}} \frac{R^{2}}{P^{2}}\left(1+\frac{P^{2}}{\mathcal{N}_{0}}\right)\right]+L^{3}\left[\frac{1}{P^{3}} \frac{R^{2}}{P}\right] \ll \frac{L^{3} M^{2}}{N^{2} P^{2}}\left(1+\frac{P^{2}}{\mathcal{N}_{0}}\right) .
\end{aligned}
$$

Lemma A.5. $\Sigma_{20} \ll(P M L)^{\varepsilon} \frac{L^{6} M^{4}}{P^{4} N^{2}}$ and $\Delta_{20} \ll(P M L)^{\varepsilon} \frac{L^{3} M^{2}}{P^{2} N}$.

Proof. The congruence conditions on $r_{1}, r_{2}$ and $n$ can be combined to write

$$
-\ell_{1} p_{2}+n r_{1} \equiv 0 \bmod p_{1} M \quad \text { and } \quad \ell_{2} p_{1}+n r_{2} \equiv 0 \bmod p_{2} M .
$$

By (22), we have $|n R| \ll N^{\varepsilon} P L<P M^{1-\varepsilon}$. The congruence conditions therefore give equalities

$$
\begin{aligned}
& n r_{1}=\ell_{1} p_{2} \quad \text { and } \quad n r_{2}=-\ell_{2} p_{1}, \\
& n^{\prime} r_{1}^{\prime}=\ell_{1} p_{2}^{\prime} \quad \text { and } \quad n^{\prime} r_{2}^{\prime}=-\ell_{2} p_{1}^{\prime} .
\end{aligned}
$$

Note that $n=\ell_{1} p_{2} / r_{1}=-\ell_{2} p_{1} / r_{2}$ implies $\ell_{1} p_{2} r_{2}=-\ell_{2} p_{1} r_{1}$. Therefore fixing $\ell_{1}, p_{2}, r_{2}$ fixes $\ell_{2}, p_{1}, r_{1}$ up to factors of $\log M$. Similarly, $n^{\prime}=\ell_{1} p_{2}^{\prime} / r_{1}^{\prime}=-\ell_{2} p_{1}^{\prime} / r_{2}^{\prime}$, so that $\ell_{1} p_{2}^{\prime} r_{2}^{\prime}=-\ell_{2} p_{1}^{\prime} r_{1}^{\prime}$. If $\ell_{1} \neq \ell_{2}$, then $\ell_{2} \mid r_{2}^{\prime}$. That saves a factor of $L$ in $r_{2}^{\prime}$ sum. Moreover for fixed $\ell_{1}, p_{2}^{\prime}, r_{2}^{\prime}$, there are only $\log M$ many $p_{1}^{\prime}, r_{1}^{\prime}$. Finally the identities $n r_{1}=\ell_{1} p_{2}$ and $n^{\prime} r_{1}^{\prime}=\ell_{1} p_{2}^{\prime}$ fix $n$ and $n^{\prime}$. 
In the case $\ell_{1}=\ell_{2}$, the previous identities become $r_{2} p_{2}=-r_{1} p_{1}$. Therefore fixing $r_{2}, p_{2}$ fixes $r_{1}, p_{1}$ up to factors of $\log M$. Finally the identity $n r_{1}=\ell p_{2}$ fixes $n$. Therefore up to a factor of $(P M L)^{\varepsilon}$,

$$
\Sigma_{20} \ll L^{5} L^{2} \frac{1}{P^{3}} R \frac{1}{P^{3}} \frac{R}{L} M^{2} \ll \frac{L^{6} M^{4}}{P^{4} N^{2}}, \quad \text { and } \quad \Delta_{20} \ll L^{3} \frac{1}{P^{3}} R M \ll \frac{L^{3} M^{2}}{P^{2} N} .
$$

Lemma A.6. $\Sigma_{21} \ll(P M L)^{\varepsilon} \frac{L^{8} M^{5}}{N^{4} P^{4}}\left(1+\frac{P^{2} M}{\mathcal{N}_{0}}\right)^{2}$ and $\Delta_{21} \ll(P M L)^{\varepsilon} \frac{L^{3} M^{5 / 2}}{N^{2} P^{2}}\left(1+\frac{P^{2} M}{\mathcal{N}_{0}}\right)$.

Proof. When $p_{1} \neq p_{2}$, the congruence $-\overline{r_{1}} \ell_{1} p_{2}+\overline{r_{2}} \ell_{2} p_{1}+n \equiv 0\left(p_{1} p_{2}\right)$ implies that $\left(n, p_{1} p_{2}\right)=1$. Moreover, for fixed $n, p_{i}$ and $\ell_{i}, i=1,2$,

$$
r_{1} \equiv \bar{n} \ell_{1} p_{2} \bmod p_{1} \quad \text { and } \quad r_{2} \equiv-\bar{n} \ell_{2} p_{1} \bmod p_{2} .
$$

These congruence conditions save a factor of $P$ in each $r_{i}$-sum. In case $p_{1}=p_{2}=p$, the congruence condition shows $p \mid n$. Moreover, $-\bar{r}_{1} \ell_{1}+\bar{r}_{2} \ell_{2}+n / p \equiv 0 \bmod p$. Therefore fixing $r_{1}, \ell_{1}, \ell_{2}, n$ saves $P$ in $r_{2}$-sum. Similarly we get saving of $P$ for each of the $n^{\prime}$ and $r_{2}^{\prime}$ sums. Also, the exact same analysis as done for $p_{i}, r_{i}, n$-sums follows for the case $\ell_{1}=\ell_{2}$. Therefore up to a factor of $(P M L)^{\varepsilon}$,

$$
\begin{aligned}
& \Sigma_{21} \ll L^{8}\left[\frac{1}{P^{2}} \frac{R^{2}}{P^{2}}\left(1+\frac{P^{2} M}{\mathcal{N}_{0}}\right) M^{1 / 2}\right]^{2}+L^{8}\left[\frac{1}{P^{2}} \frac{R^{2}}{P^{2}}\left(1+\frac{P M}{\mathcal{N}_{0}}\right) M^{1 / 2}\right]^{2} \ll \frac{L^{8} M^{5}}{N^{4} P^{4}}\left(1+\frac{P^{2} M}{\mathcal{N}_{0}}\right)^{2}, \\
& \Delta_{21} \ll L^{3}\left[\frac{1}{P^{2}} \frac{R^{2}}{P^{2}}\left(1+\frac{P^{2} M}{\mathcal{N}_{0}}\right) M^{1 / 2}\right]+L^{3}\left[\frac{1}{P^{2}} \frac{R^{2}}{P^{2}}\left(1+\frac{P M}{\mathcal{N}_{0}}\right) M^{1 / 2}\right] \ll \frac{L^{3} M^{5 / 2}}{N^{2} P^{2}}\left(1+\frac{P^{2} M}{\mathcal{N}_{0}}\right) .
\end{aligned}
$$

Acknowledgments. We are very grateful to Paul D. Nelson for many valuable comments. We are also very thankful to the referee for his or her very careful reading and detailed comments of the manuscript. K. A. and Y. L. thank their advisor R. H. for introducing them into the project and explaining the idea. Q. S. was partially supported by IRT16R43 and CSC.

\section{REFERENCES}

1. A. Adolphson and S. Sperber, Twisted exponential sums and Newton polyhedra, J. Reine Angew. Math. 443 (1993), $151-177$. MR 1241131

2. V. Blomer and G. Harcos, Hybrid bounds for twisted L-functions, J. Reine Angew. Math. 621 (2008), 53-79. MR 2431250

3. V. Blomer, G. Harcos, and Ph. Michel, A Burgess-like subconvex bound for twisted L-functions, Forum Math. 19 (2007), no. 1, 61-105, Appendix 2 by Z. Mao. MR 2296066

4. D. A. Burgess, On character sums and L-series. II, Proc. London Math. Soc. (3) 13 (1963), 524-536. MR 0148626

5. V. A. Bykovskiǔ, A trace formula for the scalar product of Hecke series and its applications, Zap. Nauchn. Sem. S.-Peterburg. Otdel. Mat. Inst. Steklov. (POMI) 226 (1996), no. Anal. Teor. Chisel i Teor. Funktsiǔ. 13, 14-36, 235-236. MR 1433344

6. J. B. Conrey and H. Iwaniec, The cubic moment of central values of automorphic L-functions, Ann. of Math. (2) 151 (2000), no. 3, 1175-1216. MR 1779567

7. W. Duke, J. Friedlander, and H. Iwaniec, Bounds for automorphic L-functions, Invent. Math. 112 (1993), no. 1, 1-8. MR 1207474

8. É. Fouvry, E. Kowalski, and Ph. Michel, Algebraic twists of modular forms and Hecke orbits, Geom. Funct. Anal. 25 (2015), no. 2, 580-657. MR 3334236

9. L. Fu, Weights of twisted exponential sums, Math. Z. 262 (2009), no. 2, 449-472. MR 2504886

10. R. Holowinsky and P. D. Nelson, Subconvex bounds on $\mathrm{GL}_{3}$ via degeneration to frequency zero, Math. Ann. 372 (2018), no. 1-2, 299-319. MR 3856814

11. E. Kowalski, Ph. Michel, and J. VanderKam, Rankin-Selberg L-functions in the level aspect, Duke Math. J. 114 (2002), no. 1, 123-191. MR 1915038

12. Y. Lin, Bounds for twists of GL(3) L-functions, arXiv:1802.05111 (2018).

13. Y. Lin, Ph. Michel, and W. Sawin, Algebraic twists of $\mathrm{GL}_{3} \times \mathrm{GL}_{2}$ L-functions, arXiv:1912.09473 (2019).

14. J. Liu, Y. Wang, and Y. Ye, A proof of Selberg's orthogonality for automorphic L-functions, Manuscripta Math. 118 (2005), no. 2, 135-149. MR 2177681

15. G. Molteni, Upper and lower bounds at $s=1$ for certain Dirichlet series with Euler product, Duke Math. J. 111 (2002), no. 1, 133-158. MR 1876443

16. R. Munshi, The circle method and bounds for L-functions-IV: Subconvexity for twists of GL(3) L-functions, Ann. of Math. (2) 182 (2015), no. 2, 617-672. MR 3418527

17. _ Twists of GL(3) L-functions, arXiv:1604.08000 (2016).

18. A note on Burgess bound, Geometry, algebra, number theory, and their information technology applications, Springer Proc. Math. Stat., vol. 251, Springer, Cham, 2018, pp. 273-289. MR 3880392 
19. I. Petrow and M. P. Young, The Weyl bound for Dirichlet L-functions of cube-free conductor, arXiv:1811.02452 (2018).

20. _ The fourth moment of Dirichlet L-functions along a coset and the Weyl bound, arXiv:1908.10346 (2019).

Department of Mathematics, The Ohio State University, 231 W 18th Avenue, Columbus, Ohio 43210-1174

E-mail address: aggarwal.78@buckeyemail.osu.edu

E-mail address: holowinsky.1@osu.edu

E-mail address: lin.1765@buckeyemail.osu.edu

School of Mathematics and Statistics, Shandong University, Weihai, Weihai, Shandong 264209, China

E-mail address: qfsun@sdu.edu.cn 